\title{
COMUNICACIÓN
}

\section{Ausencia de anfibios en bromelias de tanque en la Estación Biológica San José, Chiapas, México}

\author{
J. Manuel Aranda-Coello1,2, Oscar Miguel Mendoza Velázquez², Aarón Gómez Cruz², Joselín Cervantes Díaz², \\ Carlos Alfredo Villalobos Escobar ${ }^{2}$, Nancy Moctezuma Hérnandez ${ }^{2}$ \& Eduardo Antonio Montoya Cabrera ${ }^{2}$
}

1. Estación Biológica "San José", Laboratorio de fauna silvestre, Secretaria de Medio Ambiente e Historia Natural, Km 76.9 carretera federal libre 190, Tuxtla Gutiérrez-San Cristóbal, Municipio de Zinacantán Chiapas; m.aranda.coello@gmail.com

2. Red Mesoamericana y del Caribe para la Conservación de Anfibios y Reptiles (MesoHERP)

Recibido 20-IX-2017 • Corregido 27-XI-2017 • Aceptado 30-XI-2017

\begin{abstract}
Absence of amphibians in tank bromeliads in San José Biological Station, Chiapas, Mexico. The current population decline of amphibians, mainly due to habitat destruction and alteration by humans, is alarming. From March to May 2017, we searched for amphibians in terrestrial and arboreal bromeliads ( $>20 \mathrm{~cm}$ in height) in $16 \mathrm{ha}$ of pine-oak forest. Out of 3467 bromeliads, only two specimens had amphibians, indicating a devastating population decline.
\end{abstract}

Key words: pine-oak forest, amphibian decline, conservation, epiphytic plant, Tillandsia guatemalensis
RESUMEN: El actual declive de anfibios, principalmente por la destrucción y alteración del hábitat, por actividades antropogénicas es alarmante. De marzo a mayo del 2017, buscamos anfibios en bromelias terestres y arbóreas $(>20 \mathrm{~cm}$ de altura en 16 ha de bosque de pino-encino. De 3467 bromelias, solo dos especímenes tenían anfibios, indicando un declive devastador de la población.

Palabras claves: bosque de pino-encino, declive de anfibios, conservación, planta epifita, Tillandsia guatemalensis
Se considera que los anfibios están sufriendo la peor crisis de extinción de toda su historia (Wake \& Vredenburg, 2008). Su tasa de extinción supera a la de otros vertebrados (Collins \& Storfer, 2003; Young, Stuart, Chanson, Cox \& Boucher, 2004) y los factores identificados como causantes del declive de anfibios son de diferente índole, tanto antropogénicos tales como la destrucción de hábitat, explotación, introducción de especies exóticas, así como el efecto del cambio climático global o bien las enfermedades infecciosas emergentes (Collins \& Storfer, 2003; Daszak, Cunningham \& Hyatt, 2003; Lips, Diffendorfer, Mendelson \& Sears, 2008) y debido a sus hábitos crípticos, muchas especies se ha dificulta muestrearlas (Kays \& Allison, 2001). Los bosques de pino-encino de la estación biológica San José de Zinacatán, Chiapas. México, localizado entre las coordenadas $16^{\circ} 43^{\prime} 12^{\prime \prime} \mathrm{N}$ y $92^{\circ} 42^{\prime} 03^{\prime \prime} \mathrm{W}$, con altitudes que van desde los $2350 \mathrm{~m}$ a $2380 \mathrm{~m}$ (Mülleried, 1982) y por sus condiciones climáticas (templado subhúmeda con lluvias en verano) y su altitud representan un hábitat idóneo para alojar una gran diversidad de anfibios endémicos entre sus bromelias. Por esta razón el objetivo de nuestro estudio fue identificar la presencia de anfibios en bromelias de tanque en la estación biológica San José.

De marzo a mayo del 2017, en un área de 16ha, de bosque de pino-encino y mediante la observación directa se recorrieron transectos $\pm 700 \mathrm{~m}$ durante el día en busca de anfibios en bromelias ( $>20 \mathrm{~cm}$ de altura) tanto terrestres como arbóreas, revisando de forma manual las bromelias terrestres, y para las arbóreas, se usaron cámaras inalámbricas (GoPro Hero 3+ Silver) conectadas en tiempo real a celulares y con ayuda de bastones de $6 \mathrm{~m}$ de altura en forma de ganchos se abrían las cavidades interfóliales para observar el interior de las plantas (para obtener una mejor imagen del interior de la bromelia), como lo sugiere Aranda-Coello, Ochoa-Ochoa y Naranjo-Piñera (2012). Una vez avistado el organismo se identificaba con las claves de anfibios de Centroamérica de Köhler (2011); cada transecto era monitoreado por un 
lapso mayor a cuatro horas con un esfuerzo de muestreo de 80 horas/personas para cada transecto.

De las 3467 bromelias muestreadas únicamente se encontró en dos especies de bromelias (Tillandsia Ponderosa, T. guatemalensis) dos especies de anfibios Bolitoglossa hartwegi (la cual se considera casi amenazada (NT) por la Unión Internacional para la Conservación de la Naturaleza (IUCN, 2017) y Bolitoglossa lincolni perteneciente a la familia Plethodontidae. B. hartwegi se encontró cuatro veces, entre las primeras rosetas de T. guatemalensis (la cual estaba a nivel del suelo y con presencia de invertebrados y $1 \mathrm{~cm}$ de agua entre sus rosetas), $B$. lincolni se encontró una vez, en las primeras rosetas de $T$. ponderosa (la cual también se encontraba a nivel del suelo), aunque a diferencia de T. guatemalensis no se observó agua entre sus rosetas y estaban en proceso de descomposición.

Se reconoce que las bromelias albergan recursos alimenticios y hábitat para muchos anfibios, debido a que conforman una compleja galería entre sus rosetones, haciendo un lugar excelente para que puedan coexistir (Benzing, 1990; Beutelspacher, 1999; GalindoLeal, Cedeño-Vázquez, Calderón \& Augustine, 2003; McCracken \& Forstner, 2006; De Carvalho \& De Araújo, 2007; Mondragón Chaparro \& Cruz-Ruiz, 2009; Cruz-Ruiz, Mondragón \& Santos-Moreno, 2012; Aranda-Coello et al., 2012; García G, A., Padrón, Fernández \& Riverón-Giró, 2014) y a pesar de ello, nuestros resultados no muestran que los anfibios estén utilizando estas plantas en los bosques de pino-encino de la estación biológica y al parecer únicamente B.lincolni y $B$. hartwegi las podrían con menor frecuencia estar utilizando, lo que concuerda con lo mencionado por Parra-Olea, García-París y Wake (2004) en donde el hábitat utilizado por estas especies, es principalmente áreas cubiertas con bosque de pino-encino, en microhábitats como corteza de los troncos, debajo de piedras, en el suelo $-y$ con menor frecuencia- en bromelias arbóreas.

Desde hace unos años, el alarmante declive poblacional de anfibios, principalmente por la destrucción y alteración humana del hábitat, sumado a fenómenos naturales como las tormentas tropicales, incendios forestales y recientemente a fenómenos de gran magnitud como el sobrecalentamiento global o enfermedades emergentes pone en foco el estudio de este grupo de especies (Lips, Brem, Brenes, Reeve, Alford \& Voyles, 2006). Y al ser la estación biológica un área, que debido a su cercanía con algunas comunidades indígenas, presenta problemas ecológicos y sociales (e.g. tala ilegal, extracción de bromelias para usos tradicionales, tráfico ilegal de especies entre otros) la ausencia de anfibios podría estar relacionada con la destrucción y alteración del hábitat. Concordamos con Frías-Álvarez et al. (2010), quien determinó que el factor más importante para la disminución de las poblaciones de anfibios mexicanos es la deforestación y transformación de vegetación. Urge un estudio a fondo para determinar qué está pasando con las poblaciones de anfibios de los bosques de pino-encino de altos de Chiapas y para mitigar el problema.

\section{REFERENCIAS}

Aranda-Coello, J. M., Ochoa-Ochoa, L. M., \& Naranjo-Piñera, E. J. (2012). Evaluation of some effects of traditional harvesting of Bromeliads on the forest herpetofauna in Chanal, Chiapas. Acta Zoológica Mexicana, 28(3), 621-624.

Beutelspacher, R. (1999). Bromeliaceas como ecosistemas. México: Plaza y Valdez, S.A. de C.V.

Benzing, D. H. (1990). Vascular Epiphytes. General biology and related biota. Cambridge, Reino Unido: Cambridge University Press.

Collins, J. \& Storfer, A. (2003). Global amphibian declines: sorting the hypotheses. Diversity and Distributions, 9, 89-98.

Cruz-Ruiz, G. I., Mondragón, D., \& Santos-Moreno, A. (2012): The presence of Abronia oaxacae (Squamata: Anguidae) en tank Bromeliads en temperate forests of Oaxaca, México. Brazilian Journal of Biology, 72(2), 337-341.

Daszak, P., Cunningham, A., \& Hyatt A. D. (2003). Infectious disease and amphibian population declines. Diversity and Distributions, 9, 141-150

DeCarvalho, A.L.G., \&De Araújo, A.F.B. (2007).Ecomorphometric structure of Restinga da Marambaia lizard community, Rio de Janeiro, southeastern Brazil. Revista Brasileira de Zoología, 24(3), 786-792.

Frías-Alvarez, P., Zúñiga-Vega, J. J., \& Flores-Villela, O. (2010). A general assessment of the conservation status and decline trends of Mexican amphibians. Biodiversity and Conservation, 19, 3699-3742

Galindo-Leal, C., Cedeño-Vázquez, J. R., Calderón, R., \& Augustine, J. (2003). Arboreal frogs, tank bromeliads and disturbed seasonal tropical forest. Contemporary Herpetology, 1, 1-14.

García, G. A., Padrón, L. Y. G., Fernández, F. D., \& Riverón-Giró, F. B. (2014). Anfibios y reptiles asociados a tres especies de Bromelias de tanque en el Parque Nacional Guanahacabibes, Cuba. UNED Research Journal, 6(1), 87-97.

IUCN (Unión Internacional para la Conservación de la Naturaleza). (2017). Bolitoglossa hartwegi. Recuperado de http://www.iucnredlist.org/details/59166/0

Kays, R., \& Allison, A. (2001). Arboreal tropical forest vertebrates: current knowledge and research trends. Plant Ecology, 153, 109-120. 
Köhler, G. (2011). Amphibians of Central America. Offenbach, Germany: Verlag Elke Köhler.

Lips, K. R., Brem, F., Brenes, R., Reeve, J. D., Alford, R. A., \& Voyles, J. (2006) Emerging infectious disease and the loss of biodiversity in a neotropical amphibian community. Proceedings of the National Academy of Sciences of the United States of America, 103, 3165-3170.

Lips, K. R., Diffendorfer, J., Mendelson III, J. R., \& Sears, M. W. (2008). Riding the wave: reconciling the roles of disease and climate change in amphibian declines. PloS Biology, $6,441-454$.

McCracken, S. F., \& Forstner, M. R. (2006). Reproductive ecology and behavior of Eleutherodactylus aureolineatus (Anura, Brachycephalidae) in the canopy of the Upper Amazon Basin, Ecuador. Phyllomedusa: Journal of Herpetology, 5(2), 135-143.

Mondragón-Chaparro, D. M., \& Cruz-Ruiz, G. I. (2009). Seasonal variation of the macro-arthropod community associated to Tillandsia carlos-hankii (Bromeliaceae) in an oakpine forest in Oaxaca, Mexico. Brenesia, 70, 11-22.

Mulleried, F. K. G. (1982). La Geologia de Chiapas. 1a parte. Tuxtla, Gutiérrez, Chiapas, Mexico: Ed. Gobierno del estado de Chiapas.

Parra-Olea, G., García-París, M., \& Wake, D. B. (2004). Molecular Diversification of Salamanders of the Tropical American Genus Bolitoglossa (Caudata: Plethodontidae) and its Evolutionary and Biogeographical Implications. Biological Journal of the Linnean Society, 81, 325-346.

Wake, D., \& Vredenburg, V. (2008). Are we in midst of the sixth mass extinction? A view from the world of amphibians. Proceedings of the National Academy of Sciences, 105, 11466-11473.

Young, B., Stuart, S., Chanson, J. S., Cox, N. A., \& Boucher, T. M. (2004). Joyas que están desapareciendo: el estado de los anfibios en el Nuevo Mundo. Arlington, Virginia: Nature Serve. 